\title{
Front Matter: Volume 7639
}

, "Front Matter: Volume 7639," Proc. SPIE 7639, Advances in Resist Materials and Processing Technology XXVII, 763901 (16 April 2010); doi:

10.1117/12.863839

SPIE. Event: SPIE Advanced Lithography, 2010, San Jose, California, United States 


\section{PROCEEDINGS OF SPIE}

\section{Advances in Resist Materials and Processing Technology XXVII}

Robert D. Allen

Mark H. Somervell

Editors

22-24 February 2010

San Jose, California, United States

Sponsored by

SPIE

Cooperating Organization

SEMATECH Inc. (United States)

Published by

SPIE 
The papers included in this volume were part of the technical conference cited on the cover and title page. Papers were selected and subject to review by the editors and conference program committee. Some conference presentations may not be available for publication. The papers published in these proceedings reflect the work and thoughts of the authors and are published herein as submitted. The publisher is not responsible for the validity of the information or for any outcomes resulting from reliance thereon.

Please use the following format to cite material from this book:

Author(s), "Title of Paper," in Advances in Resist Materials and Processing Technology XXVII, edited by Robert D. Allen, Mark H. Somervell, Proceedings of SPIE Vol. 7639 (SPIE, Bellingham, WA, 2010) Article CID Number.

ISSN 0277-786X

ISBN 9780819480538

Published by

SPIE

P.O. Box 10, Bellingham, Washington 98227-0010 USA

Telephone +1 3606763290 (Pacific Time) · Fax +1 3606471445

SPIE.org

Copyright (C) 2010, Society of Photo-Optical Instrumentation Engineers

Copying of material in this book for internal or personal use, or for the internal or personal use of specific clients, beyond the fair use provisions granted by the U.S. Copyright Law is authorized by SPIE subject to payment of copying fees. The Transactional Reporting Service base fee for this volume is $\$ 18.00$ per article (or portion thereof), which should be paid directly to the Copyright Clearance Center (CCC), 222 Rosewood Drive, Danvers, MA 01923. Payment may also be made electronically through CCC Online at copyright.com. Other copying for republication, resale, advertising or promotion, or any form of systematic or multiple reproduction of any material in this book is prohibited except with permission in writing from the publisher. The CCC fee code is 0277-786X/10/\$18.00.

Printed in the United States of America.

Publication of record for individual papers is online in the SPIE Digital Library.

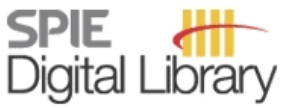

SPIEDigitalLibrary.org

Paper Numbering: Proceedings of SPIE follow an e-First publication model, with papers published first online and then in print and on CD-ROM. Papers are published as they are submitted and meet publication criteria. A unique, consistent, permanent citation identifier (CID) number is assigned to each article at the time of the first publication. Utilization of CIDs allows articles to be fully citable as soon they are published online, and connects the same identifier to all online, print, and electronic versions of the publication. SPIE uses a six-digit CID article numbering system in which:

- The first four digits correspond to the SPIE volume number.

- The last two digits indicate publication order within the volume using a Base 36 numbering system employing both numerals and letters. These two-number sets start with 00, 01, 02, 03, 04, $05,06,07,08,09,0 A, 0 B \ldots 0 Z$, followed by 10-1Z, 20-2Z, etc.

The CID number appears on each page of the manuscript. The complete citation is used on the first page, and an abbreviated version on subsequent pages. Numbers in the index correspond to the last two digits of the six-digit CID number. 


\section{Contents}

\section{Part One}

xv Conference Committee

INVITED SESSION

763902 The evolution of patterning process models in computational lithography (Invited Paper) [7639-01]

J. L. Sturtevant, Mentor Graphics Corp. (United States)

\section{DOUBLE PATTERNING AND DOUBLE EXPOSURE I}

763904 Resist material for negative tone development process [7639-03]

S. Tarutani, S. Kamimura, Y. Enomoto, K. Katou, FUJIFILM Corp. (Japan)

763905 Non-reciprocal double-exposure materials for 193nm pitch division [7639-04]

R. Bristol, J. Roberts, D. Shykind, J. M. Blackwell, Intel Corp. (United States)

763906 Photobase generator assisted pitch division [7639-05]

X. Gu, C. M. Bates, Y. Cho, T. Kawakami, T. Nagai, T. Ogata, The Univ. of Texas at Austin (United States); A. K. Sundaresan, N. J. Turro, Columbia Univ. (United States); R. Bristol, Intel Corp. (United States); P. Zimmerman, SEMATECH Inc. (United States); C. G. Willson, The Univ. of Texas at Austin (United States)

763907 The important challenge to extend spacer DP process towards 22nm and beyond [7639-06] K. Oyama, Tokyo Electron Ltd. (Japan); E. Nishimura, M. Kushibiki, Tokyo Electron AT Ltd. (Japan); K. Hasebe, S. Nakajima, H. Murakami, Tokyo Electron TOHOKU Ltd. (Japan); A. Hara, S. Yamauchi, S. Natori, K. Yabe, T. Yamaji, R. Nakatsuji, H. Yaegashi, Tokyo Electron Ltd. (Japan)

763908 Carbon-rich spin on sidewall material for self-aligned double-patterning technology [7639-07]

D. S.-H. Hsu, Nanya Technology Corp. (Taiwan); H. Yaguchi, R. Sakamoto, D. Maruyama, Y. Sakaida, Nissan Chemical Industries, Ltd. (Japan); W. Wang, C.-Y. Huang, W.-B. Wu, Nanya Technology Corp. (Taiwan); B.-C. Ho, Nissan Chemical Industries, Ltd. (Japan); C.-L. Shih, Nanya Technology Corp. (Taiwan)

EUVL: JOINT SESSION WITH CONFERENCE 7636

763909 Study on approaches for improvement of EUV-resist sensitivity [7639-08]

S. Tarutani, H. Tsubaki, H. Takahashi, T. Itou, FUJIFILM Corp. (Japan)

7639 OA Polymer photochemistry at the EUV wavelength [7639-09]

T. H. Fedynyshyn, R. B. Goodman, A. Cabral, Massachusetts Institute of Technology (United States); C. Tarrio, T. B. Lucatorto, National Institute of Standards and Technology (United States) 
7639 OB Analysis of trade-off relationships in resist patterns delineated using SFET of Selete [7639-10] T. Kozawa, Osaka Univ. (Japan); H. Oizumi, T. Itani, Semiconductor Leading Edge Technologies, Inc. (Japan); S. Tagawa, Osaka Univ. (Japan)

NOVEL RESIST MATERIALS AND PROCESSES I

7639 OC Advanced patterning solutions based on the shrink process assisted by double exposure (SPADE) [7639-11]

Y. C. Bae, Y. Liu, T. Cardolaccia, R. Bell, K. Spizuoco, G. G. Barclay, Dow Electronic Materials (United States)

7639 OD Design, synthesis, and characterization of fluorine-free PAGs for 193-nm lithography [7639-12]

S. Liu, M. Glodde, P. R. Varanasi, IBM Systems and Technology Group (United States)

7639 OE Development of an inorganic photoresist for DUV, EUV, and electron beam imaging [7639-13]

M. Trikeriotis, W. J. Bae, E. Schwartz, M. Krysak, Cornell Univ. (United States); N. Lafferty, P. Xie, B. Smith, Rochester Institute of Technology (United States); P. Zimmerman, SEMATECH Inc. (United States); C. K. Ober, E. P. Giannelis, Cornell Univ. (United States)

7639 OF A silicon-containing resist for immersion lithography [7639-14] R. Sooriyakumaran, IBM Almaden Research Ctr. (United States); W.-S. Huang, IBM Systems and Technology Group (United States); S. Swanson, H. Truong, P. Brock, A. Friz, IBM Almaden Research Ctr. (United States); K.-J. Chen, IBM Systems and Technology Group (United States); R. Allen, IBM Almaden Research Ctr. (United States)

7639 OG New self-assembly strategies for next-generation lithography [7639-15] E. L. Schwartz, Cornell Univ. (United States); J. K. Bosworth, Hitachi Global Storage Technologies, Inc. (United States); M. Y. Paik, C. K. Ober, Cornell Univ. (United States)

$7639 \mathrm{OH}$ Design and development of production-worthy developable BARCs(DBARCs) for implant lithography [7639-16]

J. Cameron, J. Amara, J. W. Sung, D. Valeri, A. Ware, K. O'Shea, Dow Electronic Materials (United States); Y. Yamamoto, H. Kitaguchi, Dow Electronic Materials (Japan); L. Vyklicky, I. Popova, P. R. Varanasi, IBM Corp. (United States)

NOVEL RESIST MATERIALS AND PROCESSES II

7639 ol Fabrication of dual damascene BEOL structures using a multilevel multiple exposure (MLME) scheme, part 1: lithographic patterning [7639-17]

D. L. Goldfarb, IBM T.J. Watson Research Ctr. (United States); S. Harrer, J. C. Arnold, IBM Research Division at Albany Nanotech (United States); S. J. Holmes, IBM Systems \& Technology Group at Albany Nanotech (United States); R. Chen, IBM Systems \& Technology Group (United States); C. Tang, N. Fender, M. Slezak, JSR Micro, Inc. (United States); R. A. Della Guardia, E. A. Joseph, S. U. Engelmann, IBM Thomas J. Watson Research Ctr. (United States); R. P. Varanasi, IBM Systems \& Technology Group (United States); M. E. Colburn, IBM Research Division at Albany Nanotech (United States) 
7639 0J Multilevel integration of patternable low-k material into advanced Cu BEOL [7639-18] Q. Lin, IBM Thomas J. Watson Research Ctr. (United States); S. T. Chen, IBM Systems and Technology Group (United States); A. Nelson, P. Brock, IBM Almaden Research Ctr. (United States); S. Cohen, IBM Thomas J. Watson Research Ctr. (United States); B. Davis, IBM Almaden Research Ctr. (United States); N. Fuller, IBM Thomas J. Watson Research Ctr. (United States); R. Kaplan, R. Kwong, IBM Systems and Technology Group (United States); E. Liniger, D. Neumayer, J. Patel, IBM Thomas J. Watson Research Ctr. (United States); H. Shobha, IBM Systems and Technology Group (United States); R. Sooriyakumaran, IBM Almaden Research Ctr. (United States); S. Purushothaman, IBM Thomas J. Watson Research Ctr. (United States); T. Spooner, IBM Corp. (United States); R. Miller, R. Allen, IBM Almaden Research Ctr. (United States); R. Wisnieff, IBM Thomas J. Watson Research Ctr. (United States)

7639 OK Advantages of BARC and photoresist matching for 193-nm photosensitive BARC applications [7639-19]

J. Lowes, Brewer Science, Inc. (United States); V. Pham, JSR Micro, Inc. (United States); J. Meador, C. Stroud, Brewer Science, Inc. (United States); F. Rosas, JSR Micro, Inc. (United States); R.-M. L. Mercado, Brewer Science, Inc. (United States); M. Slezak, JSR Micro, Inc. (United States)

$7639 \mathrm{OL}$ Sub-millisecond post exposure bake of chemically amplified resists by $\mathrm{CO}_{2}$ laser heat treatment [7639-20]

B. Jung, J. Sha, F. Paredes, C. K. Ober, M. O. Thompson, Cornell Univ. (United States); M. Chandhok, T. R. Younkin, Intel Corp. (United States)

\section{NEXT-GENERATION RESIST ARCHITECTURES}

7639 OM EUV RLS performance tradeoffs for a polymer bound PAG resist [7639-21]

R. Gronheid, A. Vaglio Pret, IMEC (Belgium); B. Rathsack, J. Hooge, S. Scheer, Tokyo Electron America, Inc. (United States); K. Nafus, H. Shite, J. Kitano, Tokyo Electron Kyushu Ltd. (Japan)

763900 Aqueous and solvent developed negative-tone molecular resists [7639-23] R. A. Lawson, J. Cheng, D. E. Noga, Georgia Institute of Technology (United States); T. R. Younkin, Intel Corp. (United States); L. M. Tolbert, C. L. Henderson, Georgia Institute of Technology (United States)

7639 OP Comparison of star and linear ArF resists [7639-24]

D. C. Forman, Cornell Univ. (United States); F. Wieberger, A. Groeschel, A. H. E. Müller, H-W. Schmidt, Univ. Bayreuth (Germany); C. K. Ober, Cornell Univ. (United States)

$76390 Q \quad$ Characteristics of main chain decomposable star shaped polymer on EUV lithography [7639-25]

T. Hirayama, J. Iwashita, S. Yoshizawa, K. Konno, T. Iwai, Tokyo Ohka Kogyo Co., Ltd. (Japan)

7639 OR Development of EUV resists based on various new materials [7639-26]

H. Oizumi, K. Matsumaro, J. Santillan, G. Shiraishi, K. Kaneyama, K. Matsunaga, T. Itani, Semiconductor Leading Edge Technologies, Inc. (Japan)

7639 OS High resolution positive-working molecular resist attached with alicyclic acid-leaving group [7639-27]

A. Yamada, S. Hattori, S. Saito, K. Asakawa, T. Koshiba, T. Nakasugi, Toshiba Corp. (Japan) 
7639 OU Negative-tone chemically amplified molecular resist based on novel fullerene derivative for nanolithography [7639-52]

H. Yamamoto, T. Kozawa, S. Tagawa, Osaka Univ. (Japan); T. Ando, K. Ohmori, M. Sato, J. Onodera, Tokyo Ohka Kogyo Co., Ltd. (Japan)

7639 OV Non-chemically amplified resists for 193-nm immersion lithography: influence of absorbance on performance [7639-53]

L. Chen, Y.-K. Goh, K. Lawrie, The Univ. of Queensland (Australia); B. Smith, Rochester Institute of Technology (United States); W. Montgomery, P. Zimmerman, SEMATECH Inc. (United States); I. Blakey, A. Whittaker, The Univ. of Queensland (Australia)

7639 OW Photobase generator and photo decomposable quencher for high-resolution photoresist applications [7639-54]

C. W. Wang, C. Y. Chang, Y. Ku, Taiwan Semiconductor Manufacturing Co. Ltd. (Taiwan)

7639 OX The optimizations of resist shrink process using track-based technology [7639-55]

Y. Kondo, A. Ookouchi, T. Tsuruda, M. Yamamoto, T. Saito, T. Shibata, S. Shimura, F. Iwao, Tokyo Electron Kyushu Ltd. (Japan); B. Rathsack, M. Carcasi, Tokyo Electron America (United States)

7639 OY Dependence of 20-nm C/H CD windows on critical process parameters [7639-56]

W.-S. Chen, P.-Y. Gu, M.-J. Tsai, Industrial Technology Research Institute (Taiwan)

$7639 \mathrm{OZ}$ Orthogonal lithography for organic electronics [7639-57]

P. G. Taylor, J.-K. Lee, A. A. Zakhidov, H. S. Hwang, J. A. DeFranco, H. H. Fong,

M. Chatzichristidi, E. Murotani, G. G. Malliaras, C. K. Ober, Cornell Univ. (United States)

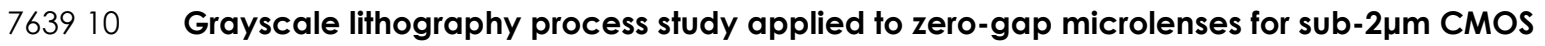
image sensors [7639-58]

S. Audran, J. Vaillant, V. Farys, F. Hirigoyen, E. Huss, B. Mortini, C. Cowache, L. Berthier, E. Mortini, J. Fantuz, O. Arnaud, L. Depoyan, F. Sundermann, C. Baron, J.-P. Reynard, STMicroelectronics (France)

763911 Improved thermal flow characteristic resist optimized for the manufacturing of microlenses [7639-59]

M. Toukhy, M. Puanescu, S. Meyer, AZ Electronic Materials USA Corp. (United States)

763912 Environmentally friendly processing of photoresists in $\mathrm{scCO}_{2}$ and decamethyltetrasiloxane [7639-60]

C. Y. Ouyang, J.-K. Lee, J. Sha, C. K. Ober, Cornell Univ. (United States)

763913 Gap-fill type HSQ/ZEP520A bilayer resist process-(IV): HSQ-rod and HSQ-tip hardening processes [7639-61]

W.-S. Chen, M.-J. Tsai, Industrial Technology Research Institute (Taiwan)

763914 LWR reduction by novel lithographic and etch techniques [7639-62]

S. Kobayashi, S. Shimura, T. Kawasaki, K. Nafus, S. Hatakeyama, H. Shite, Tokyo Electron Kyushu Ltd. (Japan); E. Nishimura, M. Kushibiki, A. Hara, Tokyo Electron AT Ltd. (Japan); R. Gronheid, A. Vaglio-Pret, IMEC (Belgium); J. Kitano, Tokyo Electron Kyushu Ltd. (Japan) 
763915 Alternatives to chemical amplification for 193nm lithography [7639-63] B. Baylav, M. Zhao, R. Yin, P. Xie, C. Scholz, B. Smith, T. Smith, Rochester Institute of Technology (United States); P. Zimmerman, SEMATECH Inc. (United States)

763917 Further analysis of the effect of point-of-use filtration on microbridging defectivity [7639-65] J. Braggin, Entegris, Inc. (United States); R. Gronheid, S. Cheng, D. Van den Heuvel,

S. Bernard, P. Foubert, IMEC (Belgium); C. Rosslee, Sokudo Co. Ltd. (United States)

763918 Point-of-use filtration methods to reduce defectivity [7639-66]

J. Braggin, Entegris, Inc. (United States); W. Schoallert, K. Hoshiko, X. Buch, JSR Micro N.V. (Belgium)

763919 Fabrication of dual damascene BEOL structures using a multilevel multiple exposure (MLME) scheme, part 2: RIE-based pattern transfer and completion of dual damascene process yielding an electrically functional via chain [7639-67]

S. Harrer, J. C. Arnold, Albany Nanotech Institute (United States); D. L. Goldfarb, IBM Thomas J. Watson Research Ctr. (United States); S. J. Holmes, Albany Nanotech Institute (United States); R. Chen, IBM Corp. (United States); C. Tang, M. Slezak, N. Fender, JSR Micro, Inc. (United States); R. A. Della Guardia, E. A. Joseph, S. U. Engelmann, IBM Thomas J. Watson Research Ctr. (United States); S.-T. Chen, D. Horak, Y. Yin, Albany Nanotech Institute (United States); R. P. Varanasi, IBM Corp. (United States); M. E. Colburn, Albany Nanotech Institute (United States)

7639 1A Resist residue removal using UV ozone treatment [7639-68]

S.-F. Chen, C.-Y. Chang, Y.-C. Ku, Taiwan Semiconductor Manufacturing Co. Ltd. (Taiwan)

7639 1B The imaging study of a novel photopolymer used in I-line negative-tone resist [7639-69] L. Liu, Y. Zou, Beijing Normal Univ. (China)

7639 1C Development of spin-on hard mask materials under resist in nano imprint lithography [7639-112]

S. Takei, Nissan Chemical Industries, Ltd. (Japan), The Univ. of Texas at Austin (United States), and Osaka Univ. (Japan); T. Ogawa, R. Deschner, K. Jen, The Univ. of Texas at Austin (United States); M. Hanabata, Nissan Chemical Industries, Ltd. (Japan); C. G. Willson, The Univ. of Texas at Austin (United States)

7639 IE Defect performance of a $2 X$ node resist with a revolutionary point-of-use filter [7639-116] J. Braggin, R. Ramirez, A. Wu, W. Choi, Entegris, Inc. (United States); I. Funahshi,

K. Yamamoto, Nihon Entegris K.K. (Japan)

7639 IF New filter rating method in practice for sub-30-nm lithography process filter [7639-117] T. Umeda, T. Mizuno, S. Tsuzuki, T. Numaguchi, Nihon Pall Ltd. (Japan)

$7639 \mathrm{1H}$ The use of surface modifiers to mitigate pattern collapse in thin film lithography [7639-1 19] D. E. Noga, W.-M. Yeh, R. A. Lawson, L. M. Tolbert, C. L. Henderson, Georgia Institute of Technology (United States)

763911 Thin film buckling as a method to explore the effect of reactive rinse treatments on the mechanical properties of resist thin films [7639-120]

W.-M. Yeh, D. E. Noga, R. A. Lawson, L. M. Tolbert, C. L. Henderson, Georgia Institute of Technology (United States) 


\section{EUV RESIST MATERIALS AND PROCESSES}

7639 1J Highly sensitive EUV-resist based on thiol-ene radical reaction [7639-70]

M. Shirai, K. Maki, H. Okamura, Osaka Prefecture Univ. (Japan); K. Kaneyama, T. Itani, Semiconductor Leading Edge Technologies, Inc. (Japan)

7639 1K Dynamics of radical cation of poly(4-hydroxystyrene) generated in thin film upon exposure to electron beam [7639-71]

K. Natsuda, T. Kozawa, K. Okamoto, A. Saeki, S. Tagawa, Osaka Univ. (Japan)

7639 IL Evaluation of hydroxyl derivatives for chemically amplified extreme ultraviolet resist [7639-72]

K. Furukawa, Y. Arai, Mitsubishi Gas Chemical Co., Inc. (Japan); H. Yamamoto, T. Kozawa,

S. Tagawa, Osaka Univ. (Japan)

7639 iN Characterization of the effects of base additives on a fullerene chemically amplified resist [7639-74]

J. Manyam, M. Manickam, J. A. Preece, R. E. Palmer, A. P. G. Robinson, The Univ. of Birmingham (United Kingdom)

\section{Part Two}

763910 Study on acid diffusion length effect with PAG-blended system and anion-bounded polymer system [7639-75]

S. Tarutani, H. Tsubaki, H. Takahashi, T. Itou, FUJIFILM Corp. (Japan); K. Matsunaga,

G. Shiraishi, T. Itani, Semiconductor Leading Edge Technologies, Inc. (Japan)

7639 IP Changes in vertical PAG distribution inside photoresist due to the variation of concentration [7639-115]

J. H. Kim, S. I. Ahn, SAMSUNG Electronics Co., Ltd. (Korea, Republic of); J. G. Yoon, POSTECH (Korea, Republic of); Y.-H. Kim, S.-K. Chae, SAMSUNG Electronics Co., Ltd. (Korea, Republic

of); W.-C. Zin, POSTECH (Korea, Republic of)

\section{DOUBLE PATTERNING AND DOUBLE EXPOSURE}

$76391 Q \quad$ Process parameter influence to negative tone development process for double patterning [7639-76]

S. Tarutani, S. Kamimura, J. Yokoyama, FUJIFILM Corp. (Japan)

7639 IR Bottom anti-reflective coatings (BARC) for LFLE double patterning process [7639-77]

R. Sakamoto, T. Endo, B.-C. Ho, S. Kimura, T. Ishida, M. Kato, N. Fujitani, R. Onishi, Y. Hiroi,

D. Maruyama, Nissan Chemical Industries, Ltd. (Japan)

7639 is Mesh patterning process for 40nm contact hole [7639-78]

K. Lee, C. Bok, J. Kim, H. Shim, J. Heo, J. Lee, H.-S. Kim, D. Yim, S.-K. Park, Hynix Semiconductor Inc. (Korea, Republic of)

7639 iT Advanced self-aligned DP process development for 22-nm node and beyond [7639-79] A. Hara, Tokyo Electron Ltd. (Japan); E. Nishimura, M. Kushibiki, Tokyo Electron AT Ltd. (Japan); S. Yamauchi, S. Natori, K. Yabe, K. Oyama, H. Yaeasghi, Tokyo Electron Ltd. (Japan) 
$76391 \mathrm{U}$ Novel approaches to controlling photo-resist CD in double patterning processes [7639-80] K. Yabe, Tokyo Electron Ltd. (Japan); K. Hasebe, S. Nakajima, H. Murakami, Tokyo Electron AT Ltd. (Japan); A. Hara, S. Yamauchi, S. Natori, K. Oyama, H. Yaeasghi, Tokyo Electron Ltd. (Japan)

7639 IV Simplified "Litho-Cluster-Only" solution for double patterning [7639-81] H. Tanaka, K. Hoshiko, T. Shimokawa, JSR Corp. (Japan); H. F. Hoefnagels, D. E. Keller, S. Wang, O. Tanriseven, R. Maas, J. Mallman, ASML Netherlands B.V. (Netherlands); K. Shigemori, C. Rosslee, SOKUDO Co., Ltd. (Japan)

7639 IW Process performance of novel resist material and novel coater/developer system for cross-line contact hole process [7639-82]

T. Nakamura, J. Yokoya, K. Ohmori, Tokyo Ohka Kogyo Co., Ltd. (Japan); H. Nakamura, T. Niwa, H. Kyouda, J. Kitano, Tokyo Electron Kyushu Ltd. (Japan)

7639 1X Improving CD uniformity for thermal cured systems in double patterning [7639-83] L. Joesten, K. Spizuoco, Y. Liu, Y. Bae, Dow Electronic Materials (United States)

7639 IY Reaction kinetics of non-reciprocal photo-base generator (NRPBG) patterning [7639-85] D. Shykind, R. Bristol, J. Roberts, J. Blackwell, Y. Borodovsky, Intel Corp. (United States)

763912 Development of reverse materials for double patterning process [7639-86]

Y. Sakaida, H. Yaguchi, R. Sakamoto, B.-C. Ho, Nissan Chemical Industries, Ltd. (Japan)

IMMERSION MATERIALS AND PROCESSING

763920 Study of post develop defect on TC-less immersion resist [7639-87]

M. Harumoto, S. Suyama, T. Miyagi, A. Hisai, M. Asai, SOKUDO Co., Ltd. (Japan)

$763921 \quad$ Novel topcoat materials with improved receding angles and dissolution properties for ArF immersion lithography [7639-88]

S. G. Yun, J. Y. Lee, Y. S. Yang, S. W. Shin, S. J. Lee, H. Y. Kwon, Y. J. Cho, S. J. Choi, S. J. Choi, J. S. Kim, T. Chang, Cheil Industries Inc. (Korea, Republic of)

763922 Evaluation of next generation hardware for lithography processing [7639-89]

T. Shimoaoki, M. Enomoto, K. Nafus, H. Marumoto, H. Kosugi, Tokyo Electron Kyushu Ltd. (Japan); J. Mallmann, R. Maas, C. Verspaget, E. van der Heijden, S. Wang, ASML Netherlands B.V. (Netherlands)

763923 Topcoat-less resist process development for contact layer of $40 \mathrm{~nm}$ node logic devices [7639-90]

M. Fujita, T. Uchiyama, NEC Electronics Corp. (Japan); T. Furusho, T. Otsuka, Tokyo Electron Kyushu Ltd. (Japan); K. Tsuchiya, Tokyo Electron Ltd. (Japan)

763924 Productivity improvement in the wafer bevel and backside cleaning before exposure [7639-91]

Y. Tokunaga, S. Nishikido, K. Yoshihara, Tokyo Electron Kyushu Ltd. (Japan); Z. Hong, Tokyo Electron Taiwan Ltd. (Taiwan); M. Strobl, Y. C. Lin, Inotera Memories, Inc. (Taiwan) 
763925 High contact angle fluorosulfonamide-based materials for immersion lithography [7639-92] D. P. Sanders, L. K. Sundberg, IBM Almaden Research Ctr. (United States); M. Fujiwara, Y. Terui, M. Yasumoto, Central Glass International, Inc. (United States)

\section{ARCS AND MULTILAYER PROCESSES}

763926 Photoresist-induced development behavior in DBARCs [7639-95]

J. D. Meador, A. Guerrero, J. A. Lowes, C. Stroud, B. Carr, A. Qin, C. Washburn,

R-M. L. Mercado, Brewer Science, Inc. (United States)

763927 Noble design of Si-SOH in trilayer resist process for sub-30-nm logic device [7639-96] T.-H. Oh, Y. Nam, C. Chang, S. Kim, M. Kwak, D. Kim, H. Shin, N.-I. Lee, J. Yoon, SAMSUNG Electronics Co., Ltd. (Korea, Republic of)

763928 Process optimization consideration for 193nm developable bottom anti-reflective coatings (DBARCs) [7639-97]

T. Kudo, S. Chakrapani, A. Dioses, E. Ng, C. Antonio, D. Parthasarathy, R. Collett, M. Neisser, M. Padmanaban, AZ Electronic Materials USA Corp. (United States)

763929 Improving material-specific dispense processes for low-defect coatings [7639-98] B. Smith, Brewer Science, Inc. (United States); R. Ramirez, J. Braggin, A. Wu, K. Anderson, Entegris, Inc. (United States); J. Berron, N. Brakensiek, C. Washburn, Brewer Science, Inc. (United States)

7639 2A Accelerating the dual damascene process time by new filling material [7639-1 14] K.-H. Tsao, Y.-H. Liu, T. Y. Chen, C.-J. Chen, C. C. Huang, United Microelectronics Corp. (Taiwan); Y.-C. Chang, G. Noya, N. Hsiao, S. Chiu, V. Chang, AZ Electronic Materials Taiwan Co., Ltd. (Taiwan); T. Katayama, H. Motobayashi, AZ Electronic Materials K.K. (Japan)

\section{MOLECULAR RESISTS}

7639 2B Development of molecular resists based on Phenyl[4]calixarene derivatives. [7639-99] M. Echigo, H. Hayashi, Mitsubishi Gas Chemical Co., Inc. (Japan); H. Oizumi, K. Matsumaro, T. Itani, Semiconductor Leading Edge Technologies, Inc. (Japan)

7639 2C All-dry processible and PAG-attached molecular glasses for improved lithographic performance [7639-100]

M. Krysak, Cornell Univ. (United States); T. Kolb, C. Neuber, H-W. Schmidt, Univ. Bayreuth (Germany); C. K. Ober, Cornell Univ. (United States)

7639 2D Molecular glass positive i-line photoresist materials containing 2,1,4-DNQ and acid labile group [7639-101]

L. Wang, J. Yu, N. XU, Beijing Normal Univ. (China)

7639 2E Novel molecular glass photoresist materials for next-generation lithography [7639-102] A. Okumura, Y. Funaki, A. Takaragi, K. Okamoto, K. Tsutsumi, K. Inoue, R. Itaya, K. Ikura, Y. Iguchi, Daicel Chemical Industries, Ltd. (Japan) 
$76392 \mathrm{~F} \quad$ Non-traditional resist designs using molecular resists: positive tone cross-linked and non-chemically amplified molecular resists [7639-103]

R. A. Lawson, D. E. Noga, J. Cheng, L. M. Tolbert, C. L. Henderson, Georgia Institute of Technology (United States)

\section{SIMULATION OF RESIST PROCESSES}

$76392 \mathrm{G}$ Three-dimensional mesoscale model for the simulation of LER in photoresists [7639-104]

R. A. Lawson, C. L. Henderson, Georgia Institute of Technology (United States)

$76392 \mathrm{H} \quad$ Evolution of resist roughness during development: stochastic simulation and dynamic scaling analysis [7639-105]

V. Constantoudis, Institute of Microelectronics (Greece); G. P. Patsis, Institute of Microelectronics (Greece) and Technological Educational Institution of Athens (Greece);

E. Gogolides, Institute of Microelectronics (Greece)

$763921 \quad$ Macroscopic and stochastic modeling approaches to pattern doubling by acid catalyzed cross-linking [7639-106]

J. Fuhrmann, A. Fiebach, Weierstrass-Institute for Applied Analysis and Stochastics

(Germany); G. P. Patsis, Technological Educational Institution of Athens (Greece)

7639 2K Extension of 248-nm Monte Carlo, mesoscale models to 193-nm plafforms [7639-108]

M. Carcasi, M. Somervell, S. Scheer, Tokyo Electron America, Inc. (United States);

S. Chauhan, J. Strahan, C. G. Willson, The Univ. of Texas at Austin (United States)

7639 2L Stochastic modeling of photoresist development in 2D and 3D [7639-109]

C. A. Mack, Lithoguru.com (United States)

$76392 \mathrm{M}$ Simulation study of directed self-assembly for 10-nm pattern formation [7639-1 10]

S.-K. Kim, H.-K. Oh, Y.-D. Jung, I. An, Hanyang Univ. (Korea, Republic of)

$76392 \mathrm{~N}$ Theoretical analysis of energy dissipation of electron in the resists II [7639-111]

M. Toriumi, Lab. for Interdisciplinary Science and Technology (Japan)

\section{RESIST FUNDAMENTALS}

763920 Methods to explore and prevent pattern collapse in thin film lithography [7639-29]

D. E. Noga, W.-M. Yeh, R. A. Lawson, L. M. Tolbert, C. L. Henderson, Georgia Institute of Technology (United States)

763925 A method to characterize pattern density effects: chemical flare and develop loading [7639-33]

L. K. Sundberg, G. M. Wallraff, A. M. Friz, IBM Almaden Research Ctr. (United States);

A. E. Zweber, Z. Benes, IBM Corp. (United States); R. D. Lovchik, E. Delamarche, IBM Research Zurich (Switzerland); W. D. Hinsberg, IBM Almaden Research Ctr. (United States)

$76392 \mathrm{~T}$ Is the resist sidewall after development isotropic or anisotropic? effects of resist sidewall morphology on LER reduction and transfer during etching [7639-34]

V. Constantoudis, G. Kokkoris, E. Gogolides, Institute of Microelectronics (Greece);

E. Pargon, M. Martin, LTM-CNRS (France) 
$76392 \mathrm{U}$ Inactivation technology for pitch doubling lithography [7639-35]

J. Hatakeyama, M. Ohashi, Y. Ohsawa, K. Katayama, Y. Kawai, Shin-Etsu Chemical Co., Ltd. (Japan)

$76392 \mathrm{~V}$ Process feasibility investigation of freezing free litho-litho-etch process for below $32 \mathrm{~nm} \mathrm{hp}$ [7639-36]

T. Nakamura, M. Takeshita, J. Yokoya, Y. Yoshii, H. Saito, R. Takasu, K. Ohmori, Tokyo Ohka

Kogyo Co., Ltd. (Japan)

$76392 \mathrm{~W} \quad$ Evolution of thermal cure resist for double patterning applications [7639-37]

M. Reilly, Y. C. Bae, V. Vohra, The Dow Chemical Company (United States); C. Koay,

M. Colburn, IBM Corp. (United States)

$76392 \mathrm{X}$ Process characterization of pitch-split resist materials for application at $16 \mathrm{~nm}$ node [7639-38]

S. J. Holmes, Albany Nanotech Institute (United States); C. Tang, JSR Micro, Inc. (United States); J. C. Arnold, IBM T. J. Watson Research Ctr. (United States); Y. Yin, Albany Nanotech Institute (United States); R. Chen, IBM Corp. (United States); N. Fender, B. Osborn, G. Dabbagh, JSR Micro, Inc. (United States); S. Liu, IBM Corp. (United States); M. Colburn, Albany Nanotech Institute (United States); R. P. Varanasi, IBM Corp. (United States); M. Slezak, JSR Micro, Inc. (United States)

$76392 Y$ Simplified double patterning process with non-topcoat self-freezing resist [7639-39] T. Fujisawa, Y. Anno, M. Hori, G. Wakamatsu, M. Mita, K. Ito, H. Tanaka, K. Hoshiko, T. Shioya, K. Goto, JSR Corp. (Japan); Y. Ogawa, H. Takikawa, Y. Kozuma, Hitachi High-Technologies Corp. (Japan); K. Fujiwara, M. Sugiura, Y. Yamaguchi, T. Shimokawa, JSR Corp. (Japan)

$76392 \mathrm{Z}$ Controlling 2D aspect ratio of elliptical contact level interconnects utilizing spin-on and reactive ion etch critical dimension shrink for the 22-nm node [7639-40] A. Metz, S. Dunn, D. Hetzer, J. Cantone, S. Kawakami, Tokyo Electron Technology Ctr., America, LLC (United States); T. Winter, Tokyo Electron America, Inc. (United States); K. Petrillo, D. Horak, S. Fan, M. Colburn, IBM Corp. (United States)

\section{SIMULATION OF LITHOGRAPHIC PHENOMENA}

763930 Roughness characterization in the frequency domain and LWR mitigation with post-litho processes [7639-41] Line-edge roughness and the ultimate limits of lithography [7639-42] C. A. Mack, Lithoguru.com (United States)

763932 Meso-scale simulation of the line-edge structure based on polymer chains in the developing and rinse process [7639-43] H. Morita, National Institute of Advanced Industrial Science and Technology (Japan); M. Doi, The Univ. of Tokyo (Japan) 
763933 Particle generation during photoresist dissolution [7639-44]

S. Chauhan, The Univ. of Texas at Austin (United States); M. Somervell, M. Carcasi, S. Scheer, Tokyo Electron America, Ltd. (United States); R. T. Bonnecaze, The Univ. of Texas at Austin (United States); C. Mack, Lithoguru.com (United States); C. G. Willson, The Univ. of Texas at Austin (United States)

763934 Predictive linewidth roughness and CDU simulation using a calibrated physical stochastic resist model [7639-45]

S. A. Robertson, J. J. Biafore, M. D. Smith, KLA-Tencor Texas (United States); M. T. Reilly,

J. Wandell, Dow Advanced Materials (United States)

763935 Reflectivity metrics for optimization of anti-reflection coatings on wafers with topography [7639-46]

M. D. Smith, T. Graves, J. Biafore, S. Robertson, KLA-Tencor Texas (United States)

\section{RESIST MATERIALS DIGEST}

763937 Continuous evolution of lithographic films through process steps: an example with 193 chemically amplified resists [7639-48]

S. Derrough, R. Tiron, CEA, LETI, MINATEC (France); D. Perret, Dow Electronic Materials, MINATEC (France); J. W. Thackeray, Dow Electronic Materials (United States); C. Sourd, CEA, LETI, MINATEC (France); P. Paniez, CNRS, LTM, MINATEC (France)

7639 3A Topcoat-less resist process for $2 \mathrm{Xnm}$ node devices [7639-51]

C. Oh, J. Lee, J. Heo, H. Shim, K. Ban, C. Bok, D. Yim, S. Park, Hynix Semiconductor Inc. (Korea, Republic of)

Author Index 
Downloaded From: https://www.spiedigitallibrary.org/conference-proceedings-of-spie on 26 Apr 2023

Terms of Use: https://www.spiedigitallibrary.org/terms-of-use 


\title{
Conference Committee
}

\author{
Symposium Chair
}

Christopher J. Progler, Photronics, Inc. (United States)

Symposium Cochair

Donis G. Flagello, Nikon Research Corporation of America (United States)

Conference Chair

Robert D. Allen, IBM Almaden Research Center (United States)

Conference Cochair

Mark H. Somervell, Tokyo Electron America, Inc. (United States)

Program Committee

Ramakrishnan Ayothi, JSR Micro, Inc. (United States)

George G. Barclay, Rohm and Haas Electronic Materials (United States)

Sean D. Burns, IBM Thomas J. Watson Research Center (United States)

Ralph R. Dammel, AZ Electronic Materials USA Corporation (United States)

Roel Gronheid, IMEC (Belgium)

Douglas J. Guerrero, Brewer Science, Inc. (United States)

Christoph K. Hohle, Fraunhofer-Center Nanoelektronische Technologien (Germany)

Scott W. Jessen, Texas Instruments Inc. (United States)

Yoshio Kawai, Shin-Ełsu Chemical Company, Ltd. (Japan)

Qinghuang Lin, IBM Thomas J. Watson Research Center (United States)

Nobuyuki N. Matsuzawa, Sony Corporation (Japan)

Vivek M. Prabhu, National Institute of Standards and Technology (United States)

Daniel P. Sanders, IBM Almaden Research Center (United States)

Thomas I. Wallow, GLOBALFOUNDRIES Inc. (United States)

Gregory M. Wallraff, IBM Almaden Research Center (United States)

Todd R. YounKin, Intel Corporation (United States)

Paul A. Zimmerman, SEMATECH Inc. (United States)

Clifford L. Henderson, Georgia Institute of Technology (United States) 


\section{Session Chairs}

1 Invited Session

Robert D. Allen, IBM Almaden Research Center (United States)

Mark H. Somervell, Tokyo Electron America, Inc. (United States)

2 Double Patterning and Double Exposure I

George G. Barclay, Dow Electronic Materials (United States)

Paul A. Zimmerman, SEMATECH Inc. (United States)

3 EUVL: Joint Session with Conference 7636

Yoshio Kawai, Shin-Etsu Chemical Company, Ltd. (Japan)

$4 \quad$ Novel Resist Materials and Processes I

Todd R. Younkin, Intel Corporation (United States)

Thomas I. Wallow, GLOBALFOUNDRIES Inc. (United States)

$5 \quad$ Novel Resist Materials and Processes II

Sean D. Burns, IBM Thomas J. Watson Research Center (United States)

Ralph R. Dammel, AZ Electronic Materials USA Corporation (United States)

6 Next-Generation Resist Architectures

Daniel P. Sanders, IBM Almaden Research Center (United States)

Roel Gronheid, IMEC (Belgium)

7 Directed Self-Assembly: Selected Semiconductor Applications: Joint Session with Conference 7637

Gregory M. Wallraff, IBM Almaden Research Center (United States)

8 Resist Fundamentals

Thomas I. Wallow, GLOBALFOUNDRIES Inc. (United States)

Todd R. Younkin, Intel Corporation (United States)

9 Double Patterning and Double Exposure II

Nobuyuki N. Matsuzawa, Sony Corporation (Japan)

Ramakrishnan Ayothi, JSR Micro, Inc. (United States)

10 Simulation of Lithographic Phenomena

Mark H. Somervell, Tokyo Electron America, Inc. (United States)

Scott W. Jessen, Texas Instruments Inc. (United States)

11 Resist Materials Digest

Qinghuang Lin, IBM Thomas J. Watson Research Center (United States)

Clifford L. Henderson, Georgia Institute of Technology (United States) 